\title{
Portending death in germinal centers - when B cells know their time is up
}

Cell Research (2018) 28:5-6. doi:10.1038/cr.2017.151; published online 1 December 2017

$B$ cells undergo stringent selection in germinal centers (GCs) for expression of high-affinity antibodies, however, mechanisms of negative selection of low-affinity B cell clones remain elusive. A new study by Michel Nussenzweig's group published in Science leverages a new reporter system that marks pre-apoptotic GC B cells to dissect microanatomic regions of GCs and their role in affinity maturation.

Our immune system faces the challenge to reliably recognize and respond to an unpredictable diversity of foreign pathogens. The antigen recognition machinery of the immune system processes the universe of antigen from bacteria and viruses in the form of peptides with an average length of ten amino acids, in principle $20^{10}$ (10.2 trillion) peptides that the immune system needs to be able to recognize. However, of the 37.2 trillion cells in the human body [1], only 0.5 trillion cells are B- and T-cells that have the capacity to express unique antigen receptor [2]. This shortfall and the fact that only a very small subset of pathogens are present at any given time is addressed by a dynamic learning and adaptation mechanism that is based on Darwinian clonal selection of lymphocytes [3]. Therefore, a very small subset of B cells are selected based on the affinity of their antibodies to their cognate antigen. From multiple rounds of genetic diversification of immunoglobulin genes by activation-induced cytidine deaminase (AID), B cells mutate and then test the affinity of their evolving antibodies. The outcome of AID-mediated mutations, which can ei- ther increase or decrease affinity, decides the fate of these B cell clones in GCs, the main site of B cell affinity maturation. In particular, elegant imaging studies have revealed important aspects of how B cells cycle through multiple rounds of proliferation, selection and cell death, as well as migrate between the two main GC compartments, the dark zone (DZ) and the light zone (LZ; illustrated in Figure 1) [4]. While B cells in the DZ (cycling, $\mathrm{CXCR} 4^{\text {high }} \mathrm{CD} 86^{\text {low }}$ ) and $\mathrm{LZ}$ (resting, $\mathrm{CXCR} 4^{\text {low }} \mathrm{CD} 86^{\text {high }}$ ) of GCs are phenotypically distinct, the contribution of these compartments to selection and affinity maturation is not clear. In addition, while GC B cells in the DZ undergo bursts of intense proliferation, the overall size of GCs and the number of GC B cells remain surprisingly stable over the course of weeks and sometimes months [5], which could be explained if rates of GC B cell apoptosis were similarly high. However, one main impediment to address these and related questions have been that functional studies involving dying cells are often problematic. Even determining the number of dying cells has been challenging due to rapid fragmentation and phagocytosis in GCs [4].

To address these limitations, Michel Nussenzweig's group has now developed transgenic and knockin mice that express a reporter (INDIA, indicator of apoptosis), which marks pre-apoptotic $B$ cells based on the cleavage of a Caspase 3 substrate [6]. Caspase 3 expression is an early irreversible event in the initiation of the apoptosis cascade. Illustrating its ability to detect pre-apoptotic GC B cells, time lapse studies showed activation of the INDIA reporter $20 \mathrm{~min}$ prior to the first signs of nuclear fragmentation and $60 \mathrm{~min}$ before the onset of cell membrane leakage. This opens up a critical time window for functional studies of cells that are fully intact yet irreversibly committed to apoptosis. Careful kinetics studies further revealed the full extent of cell death in GCs. In both DZ and LZ compartments of the GC, a small fraction of cells $(2.6 \%$ $5.7 \%$ ) were marked for apoptosis at all time points studied. This results in apoptosis of about half of all GC B cells every six hours. Despite similar rates of apoptosis, GC B cells that are dying in the DZ and LZ are dying for strikingly divergent reasons. While GC B cells in the $\mathrm{LZ}$ are destined to die, pre-apoptotic cells in the DZ were previously positively selected.

Unlike LZ B cells, $\sim 75 \%$ of preapoptotic B cells in the DZ carried AID-dependent genetic lesions that compromise their ability to express a functional antibody molecule. The extent of deleterious AID activity and its specific contribution to apoptosis in the DZ were previously unrecognized. This study reveals the main function of DZ compartment including the quality control of antibodies and elimination of B cells that were damaged by AID during fine-tuning of antibody affinity. Unlike DZ B cells, GC B cells in LZ are destined to die by default unless they are positively selected through rare random encounters with cognate T cells. Previous work highlighted CD40 ligation by Tfh cells as an essential signal driving GC B cell survival [7], however, the 


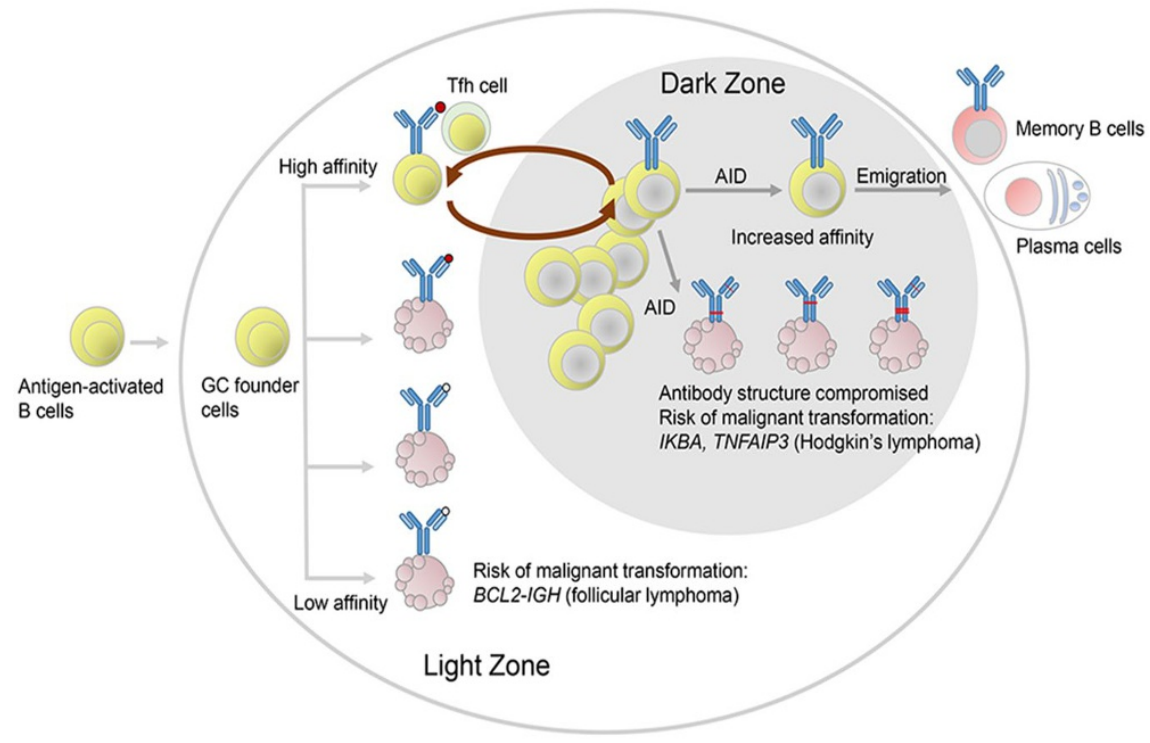

Figure 1 Fates of pre-apoptotic GC B cells. GC B cells in the light zone include highand low-affinity clones, which are selected through random encounter with $\mathrm{T}$ follicular helper (Tfh) cells. High-affinity B cells then proliferate and migrate into the dark zone. Ongoing activity of AID in the dark zone generates multiple variants including cells that lost coding capacity for a functional antibody. Other clones increase affinity, are further selected and emigrate from the GC to differentiate into long-lived memory or plasma cells.

blockade of this signal did not affect the survival of GC B cells in the LZ, suggesting that other yet unrecognized signals from Tfh cells can mediate GC B cell survival in the LZ.

Interestingly, the study provides experimental evidence that the high proliferation rate in GCs is offset by an almost equally high rate of apoptosis. This not only explains why the size of GCs remains stable for extended periods of time in the healthy state but also links the perturbations of this balance with malignant transformation as seen in different types of B cell lymphomas. In follicular lymphoma, BCL2-IGH rearrangement overrides apoptosis and causes continuous accumulation of GC B cells that may ultimately lead to lymphoma [8]. In Hodgkin's lymphoma, malignant cells express "crippled" immunoglobulin genes, which strikingly resemble AID-dependent lesions in DZ B cells [9]. The normal precursor of

destined to die. However, in Hodgkin's lymphoma, genetic lesions resulting in constitutive NF- $\mathrm{\kappa B}$ activity typically replace the survival signals that would otherwise arise from functional surface immunoglobulin [10]. To draw up a more complete scenario of malignant transformation of GC B cells, it would be important to know how long damaged GC B cells can survive until they become detectable by the INDIA reporter and subsequently die. This new experimental platform clearly has the potential to illuminate other aspects of affinity maturation in GCs that are currently unresolved. For instance, GC B cells cycle between DZ and LZ compartments and eventually emigrate from GCs to become long-lived memory or plasma cells [4], but it is not clear which factors prevent GC B cells from cycling in perpetuity and ultimately determine the number of transitions between DZ and LZ compartments. Understanding the factors that determine the rate of GC exit (up to $2 \%$ for memory B cells and $0.2 \%$ for plasma cells) and the subsequent reentry of memory B cells would yield clues for malignant transformation of GC B cells.

In addition, $\mathrm{GC}$ founder cells already exhibit a pre-apoptotic phenotype even before the onset of AID activity [11] and the INDIA reporter may be instrumental in identifying the factors that determine GC-specific propensity to cell death. Previous work identified "mitochondrial priming" reflecting apoptotic thresholds in cancer cells, as a strong predictor of drug sensitivity and resistance [12]. In similar ways, the INDIA reporter may become a powerful tool to dissect clonal evolution and enable comparative studies of drug-sensitive clones with those that have acquired drug resistance.

\section{Lili Wang ${ }^{1}$, Markus Müschen ${ }^{1}$}

${ }^{1}$ Department of Systems Biology, Beckman Research Institute and NCI City of Hope Comprehensive Cancer Center, Duarte, CA 91010, USA Correspondence: Markus Müschen E-mail: mmuschen@coh.org

\section{References}

1 Sender R, Fuchs S, Milo R. Cell 2016; 164:337-340.

2 Trepel F. Klin Wochenschr 1974; 52:511515.

3 Rajewsky K. Nature 1996; 381:751-758.

4 Allen CD, Okada T, Tang HL, et al. Science 2007; 315:528-531.

5 Robbiani DF, Deroubaix S, Feldhahn N, et al. Cell 2015; 162:727-737.

6 Mayer CT, Gazumyan A, Kara EE, et al. Science 2017; 358:pii: eaao2602.

7 Arpin C, Déchanet J, Van Kooten C, et al. Science 1995; 268:720-722.

8 Negrini M, Silini E, Kozak C, et al. Cell 1987; 49:455-463.

9 Kanzler H, Küppers R, Hansmann ML, et al. J Exp Med 1996; 184:1495-1505.

10 Jungnickel B, Staratschek-Jox A, Bräuninger A, et al. J Exp Med 2000; 191:395-402.

11 Lebecque S, de Bouteiller O, Arpin C, et al. J Exp Med 1997; 185:563-571.

12 Ni Chonghaile T, Sarosiek KA, Vo TT, et al. Science 2011; 334:1129-1133. 